\title{
A Bioengineered Implant for a Predetermined Bone Cellular Response to Loading Forces. A Literature Review and Case Report
}

\author{
Carl E. Misch, ${ }^{*}$ Martha W. Bidez, ${ }^{\dagger}$ and Mohamed Sharawy, ${ }^{\dagger}$
}

The presence of fibrous tissue has long been known to decrease the long-term survival of a root-form implant. Excessive loads on an osseointegrated implant may result in mobility of the supporting device, and excessive loads may also fracture an implant component or body. Although several conditions may cause crestal bone loss, one of these may be prosthetic overload. Excessive loads on the bone cause strain conditions to increase. These microstrains on the bone may affect the bone remodeling rate in a direct relationship.

When strain conditions to the interfacial bone are in the mild overload zone, an increased bone remodeling response occurs, which results in a reactive woven bone formation that is less mineralized and weaker. Greater stresses may cause the interfacial strain to reach the pathologic overload zone and may cause microfracture of the bone, fibrous tissue formation, and/or bone resorption. Recent reports suggest that the bone remodeling rate next to an implant may be used to evaluate biomechanical conditions and their influence on the implant-to-bone interface. These include a number of factors, such as loading conditions, implant body surface conditions, and implant design. For a given load condition, the implant design is one of the primary factors that determine the resultant strain at the interface.

A predetermined goal was established to bioengineer a dental implant to load the bone at the interface in a predetermined stress strain relationship, in order to maintain lamellar bone at the interface. A case report is presented of 2 bioengineered implants loaded for 1 year, which demonstrates that the bone was primarily lamellar in structure, the bone turnover rate was less than 5 microns/day, and was the same as the bone away from the interface. These findings corroborate those observed in a prior animal study reported with the same implant design. Although the number of implants evaluated in those 2 reports is few, they support a predetermined histological outcome. J Periodontol 2001;72:1276-1286.

\section{KEY WORDS}

Animal studies; dental implants; follow-up studies; bone and bones/anatomy and histology; bone remodeling.

Bone is an organ that responds to a number of factors including systemic changes and local mechanical forces. ${ }^{1,2}$ Wolff observed the bone adaptive properties in relation to mechanical stimuli in 1892 and proposed a hypothesis that "every change in the form and function of bone or of its function alone is followed by certain definite changes in the internal architecture, and equally definite changes in the external conformation, in accordance with mathematical laws." 3 Hence, not only does the volume of bone change when stresses are reduced (i.e., teeth are lost), but the internal organization of the remaining bone also is altered.

Cortical and trabecular bone is modified by modeling or remodeling. ${ }^{4}$ Modeling is the result of independent sites of formation and resorption, which change the shape or size of the bone. Remodeling is a process of resorption and formation at the same site that replaces previously existing bone and is primarily responsible for the change in bone quality. Bone modeling and remodeling are primarily controlled by the mechanical environment of strain.

The histologic description of bone includes lamellar bone, woven bone, composite bone, and bundle bone. The first three of these bone types are often found next to an osseointegrated dental implant. ${ }^{5}$ Composite bone is a combination of lamellar and woven bone, which forms primarily on the endosteal and periosteal surfaces of cortical bone. Lamellar bone is the most organized, highly mineralized, and strongest of the bone types; has been called "load bearing bone;" and is most desired next to an implant. ${ }^{6}$ Woven bone is also called immature bone,

\footnotetext{
* University of Michigan School of Dentistry, Department of Periodontics, Ann Arbor, MI.

$\dagger$ Department of Biomedical Engineering, University of Alabama at Birmingham, Birmingham, AL.

‡ Oral Biology/Anatomy, School of Dentistry, School of Medicine, Medical College of Georgia, Augusta, GA.
} 
since it is unorganized, less mineralized, and has less strength than the other types. These histologic terms may be used to describe the macroscopic bone types of cortical and trabecular bone.

Cortical bone fractures at levels of 10,000 to 20,000 microstrain ( $1 \%$ to $2 \%$ deformation) are dependent, in part, on the percentage of mineralization and the density of the bone. ${ }^{2}$ Nicolella et al. found that strain gauges which measured $0.15 \%$ deformation in a bone specimen may have microstructural level strain values as large as $3.5 \%$ at various regions in the microstructure. ${ }^{7,8}$ Microstrain levels 100 times less than the ultimate strength of bone may be responsible for remodeling rates within the structure, since the bone cell membranes are able to act as a mechanosensory system in bone. ${ }^{9}$ In other words, the cellular behavior of bone cells is largely determined by the mechanical environment of strain or deformation of the bone cells. ${ }^{10-12}$ It is speculated that the source of energy to open the ion membrane channels in the bone cell membrane is the microstrain in the cells as a result of the load applied to the bone. ${ }^{13,14}$

Frost described 4 microstrain zones for compact bone and related each of these categories to the mechanical adaptation of strain. ${ }^{15}$ These 4 zones include the pathologic overload zone, the mild overload zone, the adapted window, and the acute disuse window (Fig. 1). Briefly stated, the pathologic overload zone and the acute disuse window are the 2 extremes of bone reaction to strain conditions. Each of these conditions, however, may result in less bone volume. Pathologic overload could lead to microfractures, which require repair and may result in net bone resorption. The disuse zone also increases remodeling, which decreases bone mass.

\section{Frost's Bone Microstrain Zones}

\begin{tabular}{|l|l}
\hline Pathologic Overload \\
Mild Overload \\
Adapted Window \\
Acute Disuse Window \\
A.
\end{tabular}

Figure I.

The mechanostat theory of Frost ${ }^{15}$ has 4 microstrain zones for cortical bone. The acute disuse window and the pathologic overload may result in less bone. The mild overload zone results in higher bone turnover rates and more woven bone. The adapted window primarily has organized, mineralized lamellar bone.
The remodeling rate, or bone turnover, is the period of time needed for new bone to replace the existing bone and allows for the adaptation of bone to its environment, i.e., next to a dental implant. ${ }^{16}$ The bone remodeling rate (BRR) also has been expressed as a percentage or volume of new bone within a specific time period. Lamellar bone forms at a rate of 1 to 5 microns each day, while woven bone can form at rates of more than 60 microns each day. ${ }^{5}$ Hence, a higher BRR is directly related to an increase in the amount of woven bone formation. The mild overload zone is more likely to have a higher BRR than the adapted window zone and more reactive woven bone formation (less organized, less mineralized, and weaker), in order to create and maintain bone mass in response to the mechanical challenge. The adapted window zone is more likely to be organized, highly mineralized lamellar bone. In theory, the adapted window would be the ideal strain condition next to a dental implant, providing bone that is more mature and more resistant to periodic changes in strain conditions. The implant-to-bone interface in the adapted window zone would result in the BRR being similar both adjacent to and away from the implant. Therefore, the authors believe the BRR may be directly related to the strength of the implant interface and the degree of risk for the implant-bone interface. The higher risk is related to higher turnover rates, since the bone is less mineralized, less organized, and weaker at the interface.

\section{IMPLANT INTERFACE REMODELING}

Interface remodeling allows a viable bone interface to form between the dental implant and the original bone after the implant has been surgically inserted. ${ }^{5}$ By the end of 4 months of a maturation phase of bone next to a healing implant interface, osteoblasts have deposited about $70 \%$ of the mineral found in mature vital bone. The remaining 30\% of mineral deposition occurs during secondary mineralization over the next 8-month period. ${ }^{17}$ There is a direct relationship between the mineral density and the age of the bone. The greater the mineralization of bone, the stronger and stiffer the bone, and the more resistant to stresses at an implant interface. Once the bone has healed and the implant is then loaded, the interface again remodels, as influenced by its local strain environment. ${ }^{6}$ The long-term maintenance of the implant involves a continuous remodeling of the interface. In part, this allows new bone to replace bone which may have sustained microfractures and/or fatigue as a result of cyclic loading. ${ }^{15}$ In vivo microdamage in bone and an 
elevated remodeling activity to repair those regions have been identified by Frost. ${ }^{18}$ As the bone remodels, it is less mineralized at first, which also makes it weaker.

Cortical bone from the rib and/or the long bone shaft diaphysis remodels at a rate of $2 \%$ to $10 \%$ per year, ${ }^{16}$ and is similar for humans and dogs. To date, the BRR of the bone in human jaws has not yet been identified; however, in dogs it is much higher than the rib or diaphysis and approaches $40 \%$ each year. ${ }^{19}$ Hence, it is speculated that different locations in the body exhibit a different BRR that is most likely related to the microdamage resulting from repetitive loading, and the amount of microstrain in the system. Mori and Burr provided evidence of an increased BRR in regions of microfracture from fatigue damage. ${ }^{20}$ Verborgt et al. found that in the ulnae of rats, fatigue loading produced a large number of TUNEL-positive osteocytes in bone surrounding microcracks. ${ }^{21}$ The intracortical resorption was almost 300\% greater than the controls. The authors stated a strong association between microdamage, osteocyte apoptosis, and subsequent bone remodeling. Microdamage in cortical bone surrounding screw-type implants has also been reported during both insertion and with pullout forces, and the amount of microdamage was related to the thread design of the implants. ${ }^{22}$ Microdamage acts as a key step in the signaling of increases in remodeling and replacement of skeletal tissue and is similar to the local tissue remodeling in response to physical injury in other tissues (unpublished data). In addition to the increased BRR at the interface that is related to the trauma induced during implant surgery, there may be heightened remodeling of bone after loading. Hoshaw et al. found an increase in bone remodeling next to Brånemark screw implants in dog tibiae when loaded for 5 consecutive days after a 12 -month healing period. ${ }^{23}$ Other reports have also observed extensive periosteal and endosteal remodeling when pairs of screw-shaped titanium implants were constantly loaded in animal models. ${ }^{24,25}$ We believe that the increase in the BRR found in the overload zone of Frost and the increase in BRR from microfractures are directly related.

\section{MATERIALS AND METHODS}

\section{Bioengineering of an Implant Design}

It is hypothesized that if an implant design is bioengineered so that loading will produce a microstrain within the adapted window zone, it should maintain lamellar bone at the interface during loading, as represented by a similar BRR adjacent to and distal to the implant interface. Hence, a prospective goal of this implant design project involved modifying the parameters which affect the response of the surrounding bone tissue next to the implant at the cellular level. Except in the most dense bone types, endosteal dental implants are primarily surrounded by trabecular bone. Therefore, in order to design an implant body for specific strain conditions in trabecular bone, the properties of the ultimate strength and elastic modulus of trabecular bone were required to adjust the microstrain values of Frost's mechanostat conditions for cortical bone. A series of studies at the University of Alabama at Birmingham (UAB) determined the ultimate strength and modulus of elasticity of the different trabecular bone types (coarse versus fine trabeculae), along with the influence of the presence of cortical plates on these trabeculae, using fresh, frozen human specimens. ${ }^{26}$ A biomechanical method was then developed to design an implant that reduced the strain of bone at the interface, to enhance the hard tissue response by providing a lamellar bone remodeling rate around the implant interface similar to that observed several millimeters distant and to maintain that condition in response to the functional demands placed on the implant.

In order to accomplish this goal, 3 elements were first considered: 1) the macrodesign criteria were established based on a knowledge of the anatomic dimensional limitations; 2) the mechanical properties of the bone at the implant interface site were determined; and 3) the functional demands placed on the implant were assessed. Engineering principles were then applied to the geometry of the implant to determine the design features capable of enhancing strain-controlled bone turnover. The normal and shear strain equations were applied using the characterization parameters of elastic modulus and strength as input to modify the general mechanical macrodesign and thereby create a design for the implant such that the normal and shear strain on the bone-implant interface would be less than the pathologic or mild overload zones and therefore reduce the risk of bone loss or reactive woven bone. To reduce shear loading to bone, a square thread design to axial loading was used on the body of the dental implant. ${ }^{27}$ Finally, design features were refined for various regions and densities of the jaws. It was found that in order to obtain a similar microstrain in all bone densities, the square thread must be varied for each of the 4 known bone densities, ${ }^{28-30}$ in this case modifying the thread pitch and depth.

A series of patents for the concept of a bioengineered implant for optimum cellular response during 
load was applied for in 1994 by the University of Alabama at Birmingham (UAB) Research Team and eventually granted. ${ }^{31}$ This implant design was licensed by UAB to BioHorizons Implant Systems and manufactured as the Maestro implants, corresponding to each of the bone densities as described in the classifications of Lekholm and Zarb or Misch. ${ }^{28-30}$ The bone quality implant designs received U.S. Food and Drug Administration approval as the first bioengineered implant for different bone qualities in 1995. A 5-year prospective clinical trial of the bone quality bioengineered thread design§ began in June 1996. Animal and clinical studies were designed to evaluate the bioengineered implant designs in vivo.32-35 The following case report presented a unique opportunity to evaluate the BRR next to 2 of these implants loaded for 1 year in a patient.

\section{CASE REPORT}

A 35-year-old white male presented for treatment of posterior partial edentulism in May 1996. The maxillary arch was classified as Kennedy Applegate Class II, modification I (unilateral posterior missing teeth on the right region and an intrateeth edentulous space in the left posterior region). ${ }^{36}$ The patient was wearing a removable partial denture in the maxilla and presented with a dentate, restored mandibular arch (missing one first molar) and inadequate bone in height and width in the maxillary edentulous sites. He was also a skeletal Angle Class II, and orthognathic surgery and/or orthodontics was suggested to him to correct the maxillomandibular relationship. ${ }^{37}$ He declined the orthodontic treatment, but elected to follow the treatment necessary to eliminate his removable prosthesis. The patient did not live in a region that permitted postoperative follow-up evaluations for the prospective study, and therefore could not be included in the clinical trial.

In June 1996, bilateral sinus grafts with autogenous bone, alloplast, and allograft were performed in the posterior regions along with a symphysis donor graft and bone spreading in the premolar regions. In January 1997, 4 bioengineered implants $\S$ were inserted into the right posterior maxilla along with 3 implants $\S$ into the left posterior maxilla, using a 2 -stage surgical technique. All the implant bodies were $4 \mathrm{~mm}$ in diameter and 11 to $13 \mathrm{~mm}$ long.

The patient wore a removable partial denture for 7 months during the first-stage implant osseous healing. The implants were then uncovered, and the crestal bone loss observed was between 0 and $0.5 \mathrm{~mm}$. Two bilateral, fixed transitional prostheses, each completely implant supported, were fabricated and deliv- ered in August 1997 (Figs. 2, 3, and 4). Since the patient did not have any anterior occlusal contacts on natural teeth in centric relation occlusion, the implantsupported transitional prostheses were loaded in centric relation occlusion, and unilateral occlusal contacts occurred on the first premolars of the posterior prostheses during excursions.

The patient failed to continue prosthetic treatment for almost 11 months. When he finally returned for follow-up care, a request for an orthodontic consult was made, stating that the maxillary fixed prosthe-

$\S$ Maestro, BioHorizons Implant Systems, Birmingham, AL.

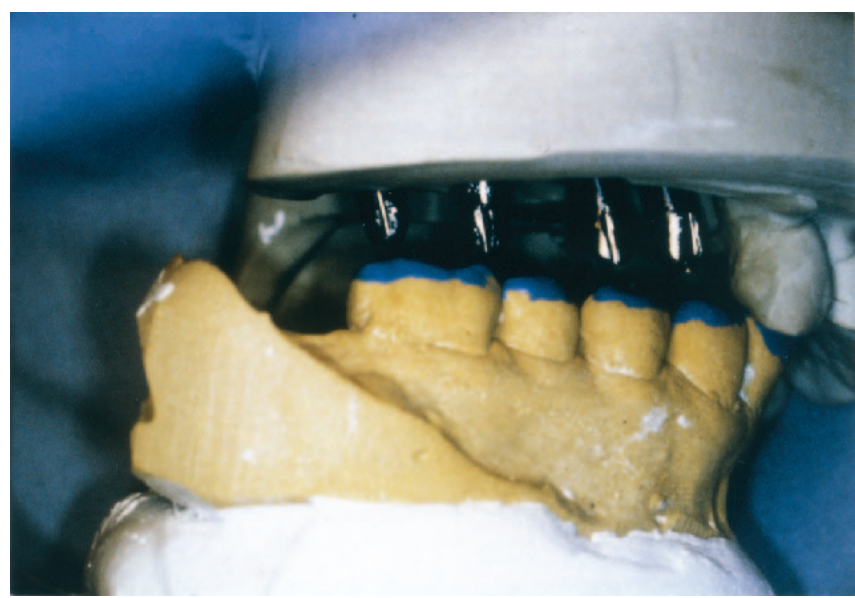

Figure 2.

Bilateral transitional prostheses were fabricated on the maxillary cast. These restorations have centric relation occlusion, and excursions occur primarily on the maxillary first premolars.

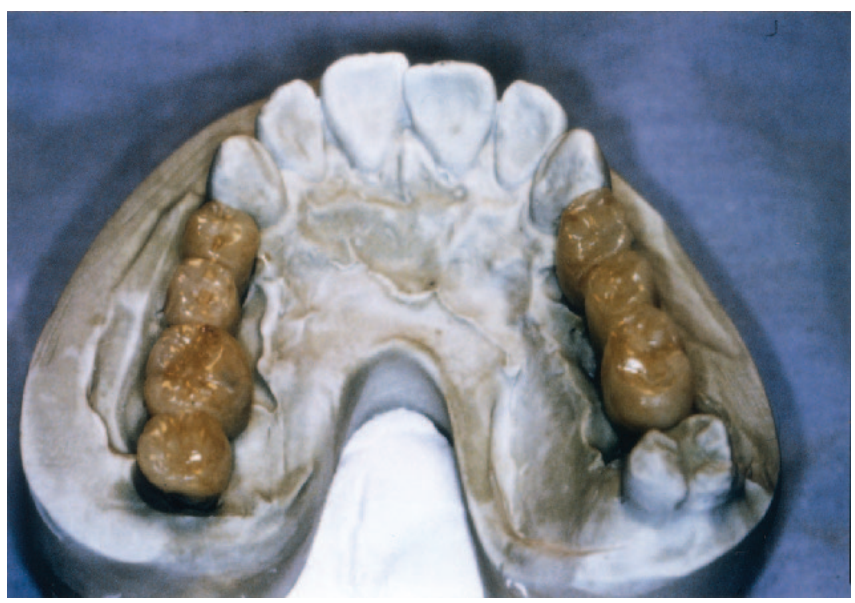

Figure 3.

The transitional prostheses in the laboratory. 


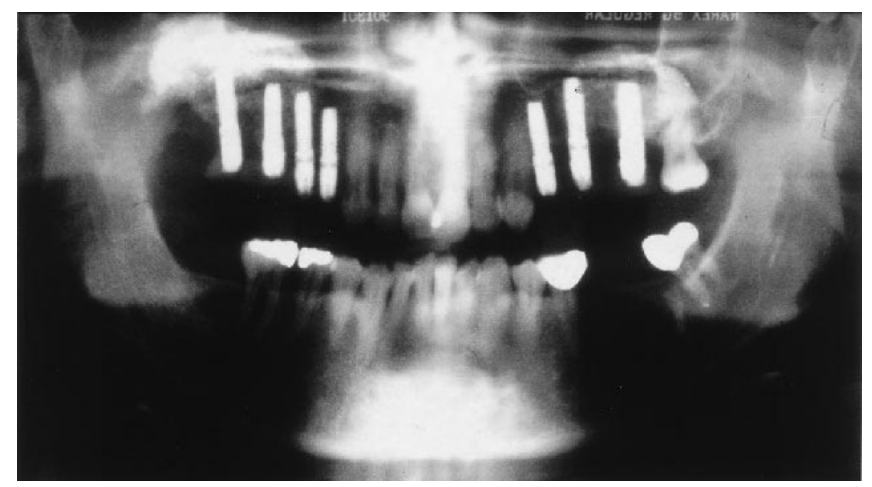

Figure 4.

A panoramic radiograph demonstrating bilateral sinus grafts, 7 dental implants in the maxillary posterior regions, and transitional restorations.

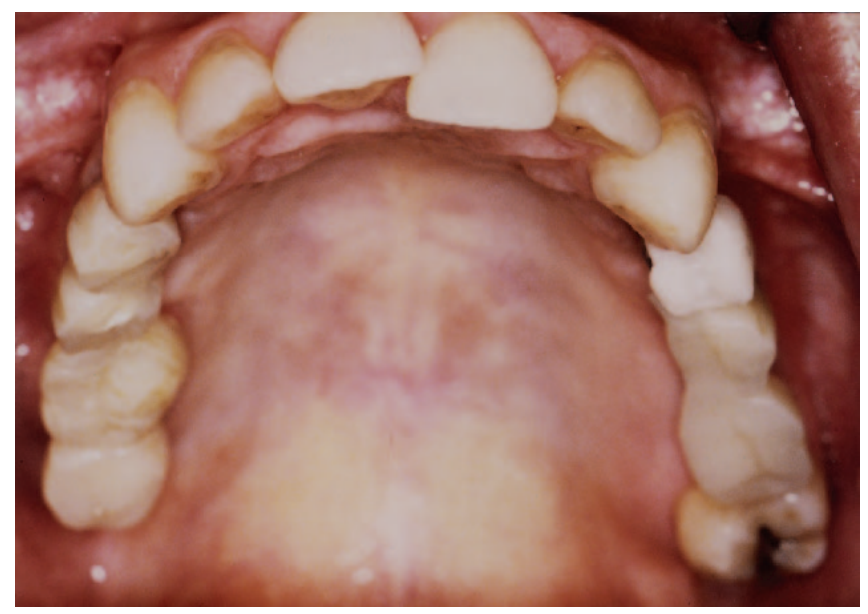

\section{Figure 5.}

The patient returned after I I months of function on the transitional prostheses. Note the severe wear related to the patient's overbite.

ses (although temporary restorations) were much better than he ever imagined, and if orthodontics could improve the appearance of his teeth, he was now willing to comply (Fig. 5). The orthodontist requested the removal of the first premolar implants in the maxilla prior to orthodontic therapy.

The patient consented to a bone labeling protocol and histologic evaluation of the retrieved implants. In order to determine the BRR more precisely next to the implants, it was decided to use 2 bone labels separated by 1 month. A bolus of tetracyline $(500 \mathrm{mg})$ was given to the patient, and 30 days later a second bolus was administered. After an additional 30 days, the implants were retrieved under copious amounts of sterile saline irrigation using a $6 \mathrm{~mm}$ end-cutting trephine bur which also removed a core of surrounding bone. The bone core and implants were placed into vials of refrigerated $10 \%$ formalin solution and sent to the Medical College of Georgia, Augusta, Georgia for histologic evaluation.

These 2 implants with attached bone were then placed in cold buffered 10\% formalin for 3 days after which they were placed in $70 \%$ alcohol until subjected to further processing. The samples were then dehydrated in ascending concentration of ethyl alcohol, up to $100 \%$, immersed into xylene, then infiltrated in methyl methacrylate (MMA) for 3 days, then MMA + benzoyl peroxide for 1 week. The samples were then embedded in fresh solution. After curing for 4 days, $500 \mu \mathrm{m}$ thick sections were cut.\| The thick sections were put on a grinder-polisher"l and thinned to $200 \mu \mathrm{m}$ and then mounted on glass slides and viewed with a fluorescent microscope." The green fluorescent lines, indicative of tetracycline labeling, were photographed. The distance between the fluorescent lines between the 2 labels was measured using special software** and a PC computer and expressed as the rate of bone formation ( $\mu \mathrm{m} /$ time period). Twelve measurements were made using 2 different sections and ranges, means, and standard errors of the means were calculated. The measurements were then divided by 30 , which represented the number of days between each bone label, which results in the $\mu \mathrm{m} /$ day of bone remodeling.

\section{RESULTS}

Concentric lamellar bone could be identified next to each of the 2 implants. Under the fluorescent microscope, 2 fluorescent lines were seen around each remodeling site. The inner label was the most recent bolus of tetracycline, while the outer label was the oldest bolus (Fig. 6). The BRR was similar for the 2 implants and was determined to average $4.866 \mu \mathrm{m}$ \pm 0.16 per day. The rate of bone formation 1 to 2 $\mathrm{mm}$ away from the outer interface was the same as the region studied within the threads. This BRR is indicative of lamellar bone remodeling. No regions of woven bone formation or significant greater remodeling rates were identified within any of the threads of either implant.

\section{DISCUSSION}

Frost has demonstrated that the bone repair process, including modeling and remodeling, is accelerated

\| Buehler Isomet 1000, Buehler, Lake Bluff, IL.

I Buehler Automet and Polisher, Buehler.

\# Zeiss, Jena, Germany.

** ImagePro, Media Cybernetics, Silver Spring, MD. 


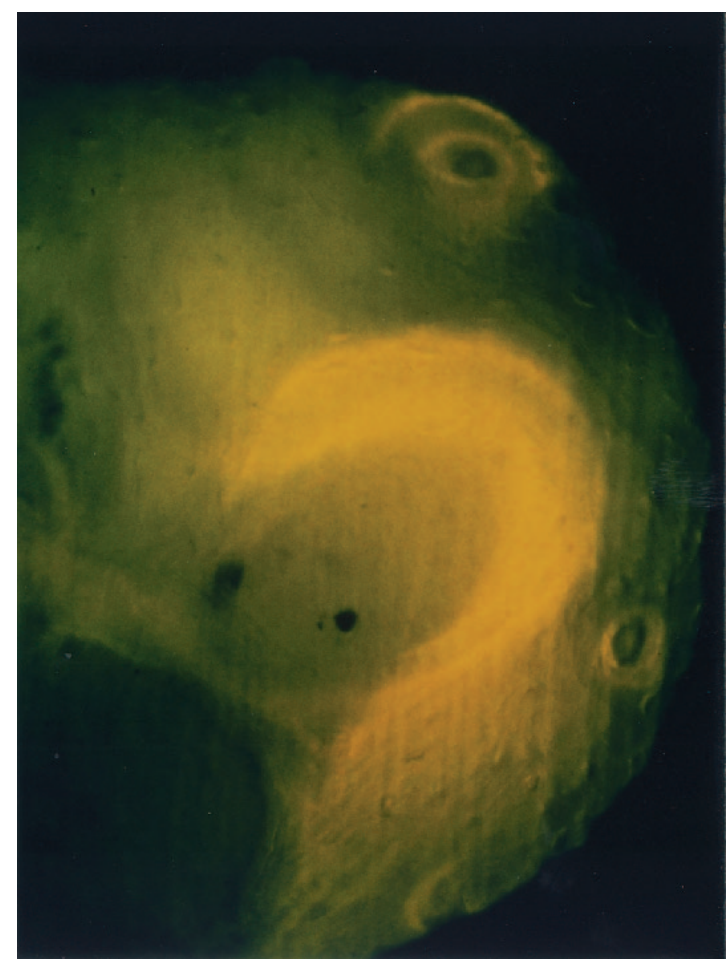

Figure 6.

The thread of a bioengineered implant in a human has 2 labels from the 2 tetracycline boli. The outer bone label represents the time of the first injection. The distance between the 2 threads is $0.5 \mathrm{~mm}$. The distance between the bone labels averaged $4.866 \mu \mathrm{m} \pm 0.16$ per day.

as a consequence of trauma (including surgery) or other noxious stimuli, and has labeled this observation as the "regional acceleratory phenomenon" (RAP). ${ }^{38}$ A major criticism of several reports that discuss observations of bone remodeling next to implants is the failure to recognize or consider this phenomenon. ${ }^{39}$ As a result, the use of unloaded control implants is highly suggested, with a similar surgical healing time frame as the loaded implants. In this trial, no control implants to evaluate the RAP influence were used. However, the case report histology is 1.5 years after the implant placement surgery, and the RAP influence, if present, should be minimal at that time frame. In addition, the RAP would increase bone remodeling. Our case report demonstrates a similar BRR adjacent to and distal from the interface.

Isidor ${ }^{40,41}$ developed a monkey model which tested the hypothesis for loss of rigid fixation from occlusal overload on implants that were successfully integrated prior to loading. Eight screw-shaped dental implants supported 4 prostheses with supra-occlusal contacts for 10 months after 8 months of bone healing in 4 ani- mals. Five of the 8 implants with supra-occlusal loads became mobile, and 1 implant lost $50 \%$ of bone. The author concluded that the failure of rigid fixation was due to fatigue microfracture in the bone around the implants, which exceeded the repair potential. Prosthetic loads to an implant-supported restoration may also cause excessive stresses, which result in fracture of implant components or even implant bodies near the crest of the bone. ${ }^{42}$ Other reports have also stated that crestal bone loss around dental implants is often related to excessive stresses which overload the bone. ${ }^{23,43,44}$ Certainly other conditions also relate to crestal bone loss, including implant design, bacteria, biologic width of implant abutment to body connection, and the density of the bone. However, excessive stresses may cause implant failure, component fracture, and/or crestal bone loss around implants.

In addition, excessive stress may also cause microfractures of bone and/or microstrains, which are in the pathologic or mild overload zone at the interface. The microstrain environment may affect the turnover rate of bone adjacent to an implant during prosthetic loading. The overload zones as described by Frost cause bone to accelerate the repair process, since regions of microfracture are more often prevalent. ${ }^{15}$ During this process, preexisting lamellar bone may become woven bone, which is less organized, less mineralized, and weaker. This reactive woven bone adjacent to an implant interface is not only weaker; it is more flexible and increases the biomechanical mismatch between the modulus of elasticity of the titanium and bone. ${ }^{26}$ This greater biomechanical mismatch increases the magnitude of strain between the bone and implant. Therefore, although the implant still exhibits rigid fixation clinically and osseointegration under the microscope, the interface bone may be more at risk of microdamage under prosthetic load because of the change in histologic structure.

Recent studies have shown that when implants act as a functionary unit for a prosthesis, an elevated BRR is an ongoing response adjacent to many dental implants. ${ }^{45}$ A BRR higher than $500 \%$ per year in the bone immediately adjacent (within $1 \mathrm{~mm}$ ) to a threaded traditional implant, but approximately 50\% in the regions 1 to $2 \mathrm{~mm}$ or more distant from the interface, has been observed. Although there is a greatly increased rate of bone remodeling supporting the dental implant, only $35 \%$ to $40 \%$ of the interface forms resorption cavities at any specific time. The remaining interface (60\% to $65 \%$ ) permits the implant to remain integrated. ${ }^{46}$ Findings by Garetto et al. 
suggest the bone at the interface of the implants in their report is likely in the mild overload zone.

The BRR may also be affected by the implant body design or surface condition. Cooper presented a histologic report of Roberts, in 1999, which evaluated the turnover rate of bone next to a macrosphere surface design implant ${ }^{\dagger \dagger}$ retrieved from a human maxilla in the premolar region. This implant had a BRR which ranged between $400 \%$ and $908 \%$ per year (unpublished data). It is interesting to note that the developers of this implant design report that crestal bone loss with smooth collar designs are often a consequence of disuse atrophy as determined by finite element analysis, rather than overload conditions. ${ }^{47}$ Hence, they believe more stress should be applied to the crestal regions. The BRR, at least in the patient reported by Cooper, appears to indicate that a greater strain condition does exist with this design, compared to other implants or conditions that have been reported. Roberts observed a difference in interface contact percentage between an asymmetric implant thread design, ${ }^{\neq} \neq$compared to a symmetrical threaded surface. ${ }^{46 \S \S}$ The V-shaped thread of the symmetrical surface $\S \S$ had a higher bone contact and reduced bone turnover rate $(500 \%)$ compared to the reverse buttress thread shape ${ }^{\ddagger \ddagger}$ (680\% BRR). Hence, the BRR reported by Roberts differs in each of these 3 differently designed implants.

Barbier and Schepers investigated implant-supported prostheses under non-axial loads and axial loads in dogs. ${ }^{48}$ Non-axial loads transmit greater stresses to bone than axial load conditions. ${ }^{49}$ Higher stresses lead to higher strain relationships in bone. A higher cellular response, including osteoblasts and inflammatory cells, was observed next to implants under non-axial shear loading conditions compared to axial loads. Barbier and Schepers also stated that non-axially loaded implants exhibited a greater BRR compared to axially loaded implants in animals with the same implant design. The remodeling activity of hydroxylapatite/tricalcium phosphate-coated (HA/ TCP) cylindrical implants has also been compared to uncoated titanium implants in rabbit tibial metaphyseal bone. ${ }^{50}$ Greater bone turnover rates were measured adjacent to titanium surface implants. This may account for the greater interface strength reported by Cook et al. ${ }^{51,52}$ with HA coatings, since greater bone mineralization (which increases the strength) occurs when the remodeling rate is reduced. Morphological changes have also been evaluated in HA-coated implants placed in humans which were extracted after functional loading had occurred for 9 to 10 years. The changes in HA morphology, com- position, and structure seemed to depend on the intensity of the stress values in the surrounding bone. ${ }^{53}$ Hoshaw found that titanium-threaded implants under axial tensile loading have higher remodeling rates and less mineralized bone than control implants that did not receive a load after healing. ${ }^{54}$

Baumgardner et al. reported an evaluation in dogs of the bone quality-based implant system presented in this report. ${ }^{32}$ After 6 months of loading with a heavy textured diet, 8 implants underwent quantitative histomorphometric analysis on the entire tissue implant interface, which demonstrated $53.7 \pm 4.2 \%$ bone contact along the entire length. Woven bone was occasionally observed, but mature formed osteons were more generally present within the threads (Fig. 7). The bone turnover rate appeared similar in the threads and in the bone several millimeters distal to the interface. The 6 -month loading period represents twice the remodeling cycle described for bone maturation in dogs, and suggests the observation period was at the point where lamellar compaction was complete. However, it is recognized that animal studies are not directly correlated to human trials, since their chewing patterns are different. Dog studies are particularly suspect since they are primarily vertical chewers, the premolars are not in occlusion, and there is an absence of bruxism or clenching. Hence, it appears the implant design, direction of load, and/or surface condition may all affect the bone at the implant interface, which affects the bone turnover rate at the interface.

The magnitude of load may also affect the BRR at the implant interface. Roberts et al. evaluated successful implants used for orthodontic anchorage for 3 years or more in humans. ${ }^{55}$ The bone-implant interface of these implants remodeled only at a rate in the range of $30 \%$ per year. Since bone in the jaws usually remodels at $40 \%$ per year, this most likely represents the adapted window zone. Although the duration of an orthodontic force is constant, the forces used for tooth movement $(<5 \text { Newtons })^{56}$ are far less than the typical forces of function or parafunction (up to 250 Newtons). As a result, the lower bone turnover rate may relate to the small magnitude of tensile loads at the implant interface during orthodontic anchorage. Brunski et al. ${ }^{57}$ attempted to use a dog model in which overloads were applied to a titanium screw dental implant. Implants in the mandibles and radii were allowed to heal for 4 to 7 months and then loaded with cyclic axial compres-

$\dagger \dagger$ Endopore, Innova Corp., Toronto, ON.

$\neq \ddagger$ Steri-Oss, Nobel Biocare, Yorba Linda, CA.

$\S \S$ Brånemark, Nobel Biocare, Gothenburg, Sweden. 


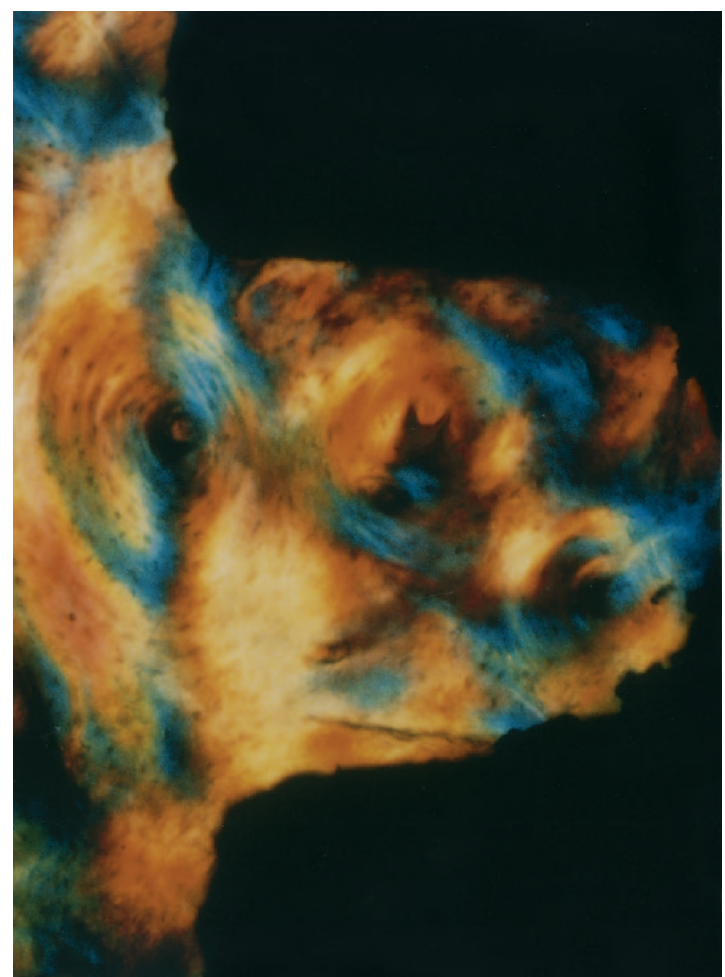

Figure 7.

A 6-month loaded bioengineered implant from a dog study. ${ }^{33}$ The presence of lamellar bone was most often found around and in the depth of the threads.

sion in the mandibles and axial tension on the radii sites. There were no significant differences between the controls and loaded interfaces. Brunski et al. believe this was most likely a result of insufficient levels of loading to observe a difference. A followup study was performed by Hoshaw et al. ${ }^{23}$ using dog tibiae and larger axial tensile loads with a longer healing time before loading. This study found more crestal bone loss around the loaded implants, an elevated BRR in the cortex, and resorptive modeling on the periosteal surface. Therefore, there are several studies and reports which demonstrate that prosthetic loading conditions on the implants can cause implant failure, ${ }^{40,44}$ crestal bone loss, ${ }^{40,43,45}$ and/or implant fracture. ${ }^{42}$ Although a few reports did not demonstrate significant differences in remodeling rates in microstrain conditions, there are many studies which related greater BRR next to implants when the load conditions place greater stresses at the interface. $22,23,44-46,48,53,55$ It is therefore hypothesized that the phenomenon of the elevated BRR at the implant interface compared to that found several millimeters away may be used as an indication of the biomechanical risk for the supporting implant-bone interface, as related or created by specific clinical conditions.

Hoshaw $^{54}$ tensile loaded titanium V-shaped threaded implants $\S \S$ in the cortical bone of canine tibiae. The osteons in the tibia are usually oriented parallel to the long axis of the bone. However, around the implants with and without axial tensile loads, osteons were oriented encircling the implants, and secondary osteons also appeared oriented around the depth of the implant threads. Hence, Hoshaw speculates that the cortical bone remodeling observed around endosteal implants may be an attempt to provide an improved strain orientation of the bone to loads.

Kohn et al. demonstrated that when the abovementioned implant $\S \S$ was laterally loaded, a bone bridge formed from the depth of one thread to another (unpublished data). Kohn et al. stated the local strain field within the bone implant interface is not homogeneous. During a lateral load, the strain is more concentrated at the point where bone contacts the outer edge of the thread, and the strain decreased from the exterior to the interior regions of the thread. They speculated that since the strains were highest at the tip of each thread, bone was resorbed, and where strains were reduced at the depth of the thread, bone was maintained. In an animal study which evaluated the implant design of this case report, microscopic observation noted that when the bone did not fully occupy the threads, greater bone volume was observed on the lower aspect, compared to the upper aspect of the square threads ${ }^{32}$ (Fig. 8). In addition, a bone bridge was found from one square thread to another. The phenomenon of bone bridging and greater bone on the inferior aspect of a threaded implant has not been previously reported under axial loads. The square thread shape of the tested implants in this report was designed to enhance compression loads and reduce shear loads delivered to the implant interface. According to Frost, the minimum effective strain for bone remodeling in hip prostheses may be larger for compressive strains. ${ }^{58}$ The occasional bone bridging observed and the difference in bone volume above and below the thread may be related to the mechanism of load transfer on the bone from the square thread design. Therefore, the orientation of the osteons around implant threads, the bone bridging findings of the square thread, the report of Kohn et al., and the observation of more bone on the inferior aspects of the square implant threads with axial loads may all be related to different strain regions of the threads. 


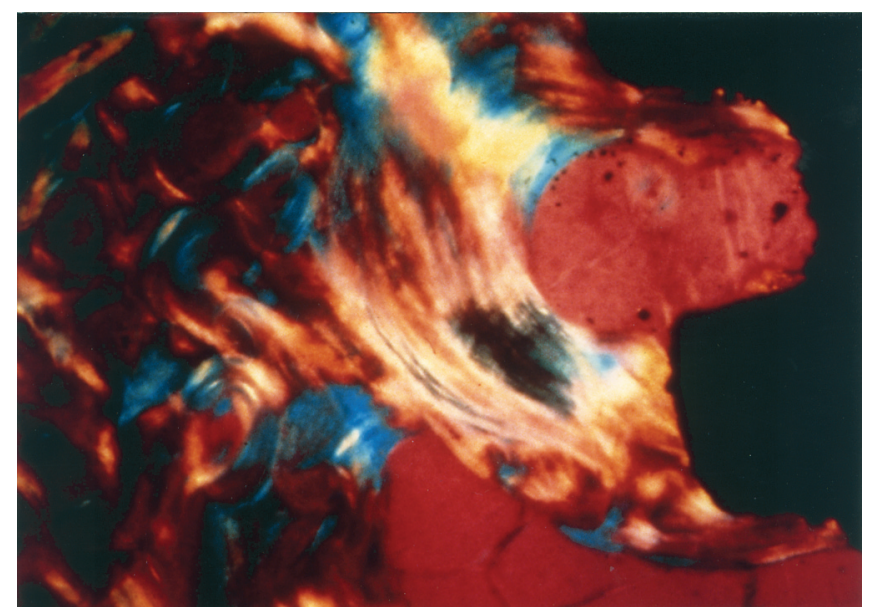

Figure 8.

Bone bridging from one thread to another was occasionally seen. This phenomenon has not previously been reported with implants loaded in their long axis. In general, if bone did not occupy the entire space of the threads, more bone was found on the inferior, compared to the superior, aspect of the square thread.

A hypothesis to bioengineer a dental implant for a predetermined bone cellular response to occlusal loads was established prior to clinical investigation by our group. The desired histologic condition was a reduced BRR next to a functioning dental implant. Although data were provided by only one human patient, the determination of bone formation/day next to the test implant design reported is significant, since it observes a histologic condition established at the onset of the engineering process. The turnover rate of bone within the threads of the implants $\S$ in this report was less than 5 microns/day, and was the same as observed in the bone away from the implant interface. This rate corresponds to the rate of lamellar bone remodeling in the adapted window zone. This condition may place the interface at less biomechanical risk, since lamellar bone is more mineralized, more rigid, and stronger compared to the reactive woven bone found in the mild overload zone.

\section{CONCLUSIONS}

The remodeling rate, or bone turnover, is the period of time for new bone to replace the existing bone and allows for adaption of bone to its environment. Bone turnover rates have been measured around healing implants. These remodeling rates have also been observed around short-term loaded implants with different surface conditions (i.e., hydroxylapatite versus titanium), designs (i.e., Nobel Biocare versus Endo- pore), and different loading conditions (axial versus offset loading). Bone strength and modulus of elasticity are related to its microscopic structure. For example, woven bone is weaker and more flexible than lamellar bone. Weaker bone may microfracture more easily, and more flexible bone has a greater biomechanical mismatch to a titanium implant, which increases the strain condition at the interface. The bone remodeling rate (BRR) influences the amount of woven bone surrounding a loaded dental implant. Hence, the authors believe BRR may be used to evaluate risk factors related to stress and/or strain at the cellular level and may be used in the future as a method to evaluate and compare the biomechanical risks related to long-term implant success. An implant with a reduced BRR is more likely to maintain lamellar bone at the interface and, in theory, is advantageous for long-term implant survival and osseous maintenance.

This paper presents a concept that employs the microstrain zones for bone described by Frost ${ }^{15}$ to develop a bioengineered dental implant which is more likely to maintain a lamellar bone interface under loading conditions. As part of this project, the mechanical properties of trabecular bone and the influence of the cortical plates were first determined; then, using finite element analysis, an implant design was created for each bone density to microstrain the bone within the adapted window zone. An animal study followed, which demonstrated a similar BRR within the bone threads and adjacent to the implant. A human case report is presented, which also demonstrated a BRR similar to the surrounding trabecular bone, which was reduced compared to several reports in the literature. Hence, the prospective goal of designing a bioengineered implant that may produce a BRR within the threads similar to the lamellar surrounding bone appears possible.

Further documentation is required under several different clinical situations to confirm (or deny) the findings in this report. The concept presented in this paper is not meant to suggest that adequate strain levels may only be obtained with this implant design, or even that ideal strain levels are necessary for longterm maintenance of osseointegration. The strain environment around an endosteal implant is very complex, and the variables of bone density, bone volume, and bone shape are most likely influential, yet not understood. Future experimental models should evaluate how the influence of implant design, surface condition, intensity of load, frequency of load, direction of force, and bone density interrelate to the longterm success of dental implants. It is suggested that 
the bone turnover rate may be a method to evaluate these conditions.

\section{ACKNOWLEDGMENTS}

This project was supported by the University of Alabama at Birmingham (UAB) and its spin-out company, BioHorizons Implant Systems. Carl E. Misch and Martha W. Bidez served as codirectors for UAB with this project and are members of the Board of Directors and consultants to BioHorizons. Together, they hold less than 3\% of the stock in this company.

\section{REFERENCES}

1. Rhinelander FW. Circulation in bone. In: Bourne GH, ed. The Biochemistry and Physiology of Bone. New York: Academic Press; 1972:1-77.

2. Currey JD. The Mechanical Adaptations of Bones. Princeton: Princeton University Press; 1984:1-294.

3. Wolff J. Principles of Bone Transformation, translated by Hujust Hurschwald. Berlin, 1892.

4. Enlow DH. Principles of Bone Remodeling. Springfield, IL: Charles C. Thomas; 1963.

5. Roberts WE, Turley PK, Brezniak N, Fielder PJ. Bone physiology and metabolism. Calif Dent Assoc J 1987; 15:54-61.

6. Roberts WE, Smith RK, Zilerman Y, Mozary PG, Smith RS. Osseous adaptation to continuous loading of rigid endosseous implants. Am J Orthod 1984;86:95-111.

7. Nicolella DP, Lankford J, Jepsen K, Davy DT. Correlation of physical damage development with microstructure and strain localization in bone. In. Chandrin KB, Vanderby R Jr., Hefzy MS, eds. Bioengineering Conference, vol. 35. New York: American Society of Mechanical Engineers, 1997:311-312.

8. Nicolella DP, Nicholls AE, Lankford J. Micromechanics of Creep in Cortical Bone. Chicago: Orthopedic Research Society; 1998:137.

9. Cowin SC, Moss-Salentyin L, Moss MC. Candidates for mechanosensory system in bone. J Biomech Engineer 1991;113:191-197.

10. Rubin CT, Hausman MR. The cellular basis of Wolff's law. Transduction of physical stimuli to skeletal adaption. Rheum Dis Clin North Am 1988;14:503-517.

11. Brighton CT, Strafford B, Gross SB, Leatherwood DF, Williams JL, Pollack SR. The proliferative and synthetic response of isolated calvarial bone cells of rats to cyclic biaxial mechanical strain. J Bone Joint Surg 1991;73: 320-331.

12. Jones DB, Nolte H, Scholubbers JG, Turner E, Veltel D. Biomechanical signal transduction of mechanical strain in osteoblast like cells. Biomaterials 1991;12:101110.

13. Sachs F. Mechanical transduction in biological systems. Crit Rev Biomed Eng 1988;16:141-169.

14. Sachs F. Mechanical transduction by membrane ion channels: A mini review. Mol Cell Biochem 1991;104: 57-60.

15. Frost HM. Mechanical adaptation. Frost's mechanostat theory. In: Martin RB, Burr DB, eds. Structure, Function and Adaption of Compact Bone. New York: Raven
Press; 1989:179-181.

16. Parfitt $\mathrm{AM}$. The physiological and clinical significance of bone histomorphometric data. In Reck RR, ed. Bone Histomorphometry, Techniques and Interpretation. Boca Raton, FL: CRC Press; 1983:143-223.

17. Roberts WE, Garetto LP, De Castro RA. Remodeling of devitalized bone threatens periosteal margin integrity of endosseous titanium implants with threaded or smooth surfaces: Indications for provisional loading and axially directed occlusion. J Indiana Dent Assoc 1989;68:19-24.

18. Frost HM. Presence of microscopic cracks in vivo in bone. Henry Ford Hosp Med Bull 1960;8:25-35.

19. Tricker ND, Garetto LP. Cortical bone turnover and mineral apposition in dentate dog mandible. J Dent Res 1977;76(Spec. Issue):201.

20. Mori S, Burr DB. Increased intracortical remodeling following fatigue damage. Bone 1993;14:103-109.

21. Verborgt O, Gibson GJ, Schaffler MB. Loss of osteocyte integrity in association with microdamage and bone remodeling after fatigue in vivo. $J$ Bone Miner Res 2000;15:60-67.

22. Hoshaw SJ, Schaffler MB, Fyhrie DP. Effect of thread design on microdamage creation in cortical bone. Trans Orthopaed Res Soc 1994;19:537.

23. Hoshaw SJ, Brunski JB, Cochran GVB. Mechanical loading of Branemark fixtures affects interfacial bone modeling and remodeling. Int $J$ Oral Maxillofac Implants 1994;9:345-360.

24. Lufferts R, Turley PK. Force application to bioglass coated alumina implants of various sizes. J Dent Res 1982;61(Spec. Issue):339(Abstr. 1447).

25. Ogiso B, Hashimoto M, Valiquette N, Carter S, Pilliar RM. A rat model for studying mechanical forces around implants. J Dent Res 1991;70(Spec. Issue):400(Abstr. 1072).

26. Misch CE, Qu M, Bidez MW. Mechanical properties of trabecular bone in the human mandible: Implications for dental implant treatment planning and surgical placement. J Oral Maxillofac Surg 1999;57:700-706.

27. Misch CE, Bidez MW. A Scientific rationale for dental implant design. In: CE Misch, ed. Contemporary Implant Dentistry, 2nd ed. St. Louis: Mosby; 1999:329343.

28. Lekholm U, Zarb GA. Patient selection and preparation. In: Branemark PI, Zarb GA, Albreksson T, eds. Tissue Integrated Prostheses: Osseointegration in Clinical Dentistry. Chicago: Quintessence; 1985:199-209.

29. Misch CE. Bone character: Second vital implant criterion. Dent Today 1988;7:39-40.

30. Misch CE. Density of bone effect on treatment plans, surgical approach, healing and progressive loading. Int $J$ Oral Implant 1990;6:23-31.

31. Misch CE, Bidez MW, Strong JT. Design process for skeletal implants to optimize cellular response. US patent 5,628,630. May 13, 1997.

32. Baumgardner JD, Boring G, Cooper RC, et al. Preliminary evaluation of a new dental implant design in canine models. Implant Dent 2000;9:252-260.

33. Misch CE, Hoar JE, Beck G, Hazen R, Misch CM. A bone quality-based implant system: a preliminary report of stage I and stage II. Implant Dent 1998;7:35-41. 
34. Hoar JE, Beck GH, Crawford EA, Resnik R. Prospective evaluation of crestal bone remodeling of a bone density-based dental implant system. Compendium Continuing Educ Dent 1998;19:17-24.

35. Misch CE, Dietsh-Misch F, Hoar J, et al.. A bone quality-based implant system (BioHorizons Maestro Dental Implants): A prospective study of the first year of prosthetic loading. J Oral Implant 1999;25:185-197.

36. Kennedy E. Partial Denture Construction. Brooklyn, NY: Dental Items of Interest Publishing Co.; 1925:2325.

37. Angle EH. Malocclusion of the Teeth, 7th ed. Philadelphia: SS White Dental Mfg. Co.; 1907.

38. Frost HM. The regional acceleratory phenomenon: A review. Henry Ford Hosp Med Bull 1983;31:3-9.

39. Brunski JB, Puleo DA, Nanci A. Biomaterials and biomechanics of oral and maxillofacial implants: Current status and future developments. Int $J$ Oral Maxillofac Implants 2000;15:15-46.

40. Isidor F. Loss of osseointegration caused by occlusal load of oral implants. A clinical and radiographical study in monkeys. Clin Oral Implants Res 1996;7:143-152.

41. Isidor F. Histological evaluation of peri-implant bone at implants subjected to occlusal overload or plaque accumulation. Clin Oral Implants Res 1997;8:1-9.

42. Rangert B, Krogh-PHJ, Langon B, van Roekel N. Bending overload and implant fracture. A retrospective clinical analysis. Int J Oral Maxillofac Implants 1995;10: 326-334.

43. Misch CE. Early crestal bone loss etiology and its effect on treatment planning for implants. Postgrad Dent 1995;2(3):3-17.

44. Quirynen M, Naert F, van Steenberghe D. Fixture design and overload influence in marginal bone loss and fixture success in the Branemark implant system. Clin Oral Implants Res 1992;3:104-111.

45. Garetto LP, Chen J, Parr JA, Roberts WE. Remodeling dynamics of bone supporting rigidly fixed titanium implants: A histomorphometric comparison in four species including humans. Implant Dent 1995;4:235243.

46. Roberts WE. Bone dynamics of osseointegration, ankylosis, and tooth movement. J Indiana Dent Assoc 1997; 78:24-32

47. Vaillancourt H, Pilliar RM, McCammond D. Finite element analysis of crestal bone loss around porous-coated dental implants. J Appl Biomater 1995;6:267-282.
48. Barbier L, Schepers E. Adaptive bone remodeling around oral implants under axial and non axial loading conditions in the dog mandible. Int $J$ Oral Maxillofac Implants 1997;12:215-223.

49. Misch CE, Bidez MW. Implant protected occlusion: A biomechanical rationale. Compendium Continuing Educ Dent 1994;15:1330-1342.

50. Burr DB, Mori S, Boyd RD, et al. Histomorphometric assessment of the mechanics for rapid ingrowth of bone to HA/TCP coated implants. J Biomed Mater Res 1993;27:645-653.

51. Cook SD, Kay JF, Thomas KA, Jarcho M. Interface mechanics and histology of titanium and HA coated titanium for dental implant applications. Int J Oral Maxillofac Implants 1987;2:15-22.

52. Thomas KA, Kay JF, Cook SD, Jarcho M. The effect of surface macrotexture and hydroxylapatite coating on the mechanical strengths and histologic profiles of titanium implant materials. J Biomed Mater Res 1987;21: 1395-1414.

53. Baltag I, Watanabe K, Kusakari H, et al. Long-term changes in hydroxylapatite coated dental implants. $J$ Biomed Mater Res 2000;53:76-85.

54. Hoshaw SJ. Investigation of bone modeling and remodeling at a loaded bone-implant interface. [Thesis]. Troy, NY: Rensselaer Polytechnic Institute; 1992.

55. Roberts WE, Marshall KJ, Mosary PG. Rigid endosseous implants utilized as anchorage to protract molars and close an atrophic extraction site. Angle Orthod 1990; 60:135-152.

56. Roberts WE, Halm FR, Marshall KJ, Gongloff RK. Rigid endosseous implants for orthodontic and orthopedic anchorage. Angle Orthod 1989;59:247-256.

57. Brunski JD, Hipp JA, Cochran GVB. The influence of biomechanical factors at the tissue-biomaterial interface. In: Hanker JS, Girnmara BC, eds. Biomechanical Materials and Devices. Pittsburgh: Materials Research Society; 1989:505-515.

58. Frost HM. Some design problems in hip replacement prostheses: An analysis, intermediary organization of the skeleton. Boca Raton, FL: CRC Press; 1986:221-241.

Send reprint requests to: Dr. Carl E. Misch, 16231 W. Fourteen Mile Road, Beverly Hills, MI 48025. Fax: 248/6423794; e-mail: info@misch.com

Accepted for publication March 16, 2001. 